Draft VERSion OCTOBER 15, 2018

Preprint typeset using $\mathrm{IAT}_{\mathrm{E}} \mathrm{X}$ style emulateapj v. 5/2/11

\title{
DOES THE SAGITTARIUS STREAM CONSTRAIN THE MILKY WAY HALO TO BE TRIAXIAL?
}

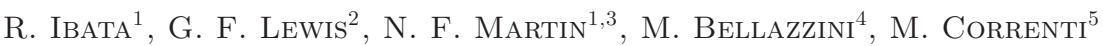 \\ Draft version October 15, 2018
}

\begin{abstract}
Recent analyses of the stellar stream of the Sagittarius dwarf galaxy have claimed that the kinematics and three-dimensional location of the M-giant stars in this structure constrain the dark matter halo of our Galaxy to possess a triaxial shape that is extremely flattened, being essentially an oblate ellipsoid oriented perpendicular to the Galactic disk. Using a new stream-fitting algorithm, based on a Markov Chain Monte Carlo procedure, we investigate whether this claim remains valid if we allow the density profile of the Milky Way halo greater freedom. We find stream solutions that fit the leading and trailing arms of this structure even in a spherical halo, although this would need a rising Galactic rotation curve at large Galactocentric radius. However, the required rotation curve is not ruled out by current constraints. It appears therefore that for the Milky Way, halo triaxiality, despite its strong theoretical motivation, is not required to explain the Sagittarius stream. This degeneracy between triaxiality and the halo density profile suggests that in future endeavors to model this structure, it will be advantageous to relax the strict analytic density profiles that have been used to date.
\end{abstract}

Subject headings: Galaxy: halo — dark matter — Galaxies: dwarf

\section{INTRODUCTION}

In standard $\Lambda$ Cold Dark Matter $(\Lambda$-CDM) cosmology Komatsu et al. 2011) galaxies form within dark matter halos that coalesce through repeated mergers. High resolution pure CDM simulations give rise to significantly triaxial galaxy halos, that become progressively less triaxial towards lower sizes and masses due to the increased dynamical age of the lower mass structures Allgood et al. 2006). The inclusion of baryons in galaxy formation simulations alters dramatically the behavior of the central regions of the halo, which added to the effect of merging satellites makes the central halo become rounder (Debattista et al. 2008).

In the Milky Way, we have perhaps the best opportunity to constrain the shape of any dark matter distribution. One particularly promising strategy to accomplish this, and which is generally not possible in extragalactic systems, is to use streams of stars to probe the global potential. The streams of low-mass satellites follow fairly closely the centre of mass orbit of their progenitor, and with careful modeling it is possible to find a solution for the stream structure within a Galactic mass distribution. This approach has been used in the past to model the stream of the Sagittarius (Sgr) dwarf galaxy (Ibata et al. 2001), probably the most significant accretion into the Milky Way in the last $\sim 5$ Gyr. Recently, Law \& Majewski (2010, hereafter LM10) presented a new analysis of the spatial and kinematic structure of Mgiant star members of this stream (Majewski et al. 2003, 2004), and showed that their data could be reproduced

\footnotetext{
${ }^{1}$ Observatoire Astronomique, Université de Strasbourg, CNRS, 11, rue de l'Université, F-67000 Strasbourg, France

2 Sydney Institute for Astronomy, School of Physics, A28, The University of Sydney, NSW 2006, Australia

${ }_{3}$ Max-Planck-Institut für Astronomie, Königstuhl 17, 69117 Heidelberg, Germany

${ }^{4}$ INAF-Bologna Astronomical Observatory, via Ranzani 1, I40127 Bologna, Italy

${ }^{5}$ INAF-Istituto di Astrofisica Spaziale e Fisica Cosmica, Via P. Gobetti, 101, I-40129 Bologna, Italy
}

if the dark halo of the Milky Way has a triaxial form with a minor to major axis ratio of $(c / a)_{\Phi}=0.72$ and intermediate to major axis ratio of $(b / a)_{\Phi}=0.99$ (in the potential). The structure is therefore almost an oblate ellipsoid, but with its minor axis contained within the Galactic plane, which presumably induces non-circular orbits at radii where the disk is not dominant. As LM10 point out, such a halo is not natural in $\Lambda$-CDM. It is also worth noting that their model has an extremely high degree of oblateness in density. These conclusions have been further reinforced by the analysis of Deg \& Widrow (2012, hereafter DW12) who in fitting the stream with orbits in a Galactic mass model, found a similar oblate dark matter halo also oriented perpendicular to the Galactic plane. In this contribution we aim to determine whether it is possible to reproduce the structure and kinematics of the Sgr stream, at least as well as LM10, without invoking a triaxial Galactic halo.

\section{MODELLING}

One means to model the formation of the Sgr stream would be to make a mass model of the Milky Way and an N-body model of the Sgr dwarf, and to evolve the latter inside the potential provided by the former using an N-body integrator. Through a judicious sampling of orbital and structural parameters of the two bodies it would be possible to assess the triaxiality issue. This is essentially the strategy adopted by LM10, but it has the disadvantage of being computationally extremely costly, which greatly limits the resolution of the parameter space survey. An alternative approach, adopted by DW12, is to follow orbits in a mass distribution, however due to self-gravity this is not a good approximation for streams of massive satellites (Varghese et al. 2011).

Instead, we have chosen to use a new stream-fitting algorithm presented in Varghese et al. (2011), which searches through the parameter space of the host galaxy mass model and the satellite's orbital properties and mass using a Markov Chain Monte Carlo (MCMC) scheme. As we have shown in that contribution, the 

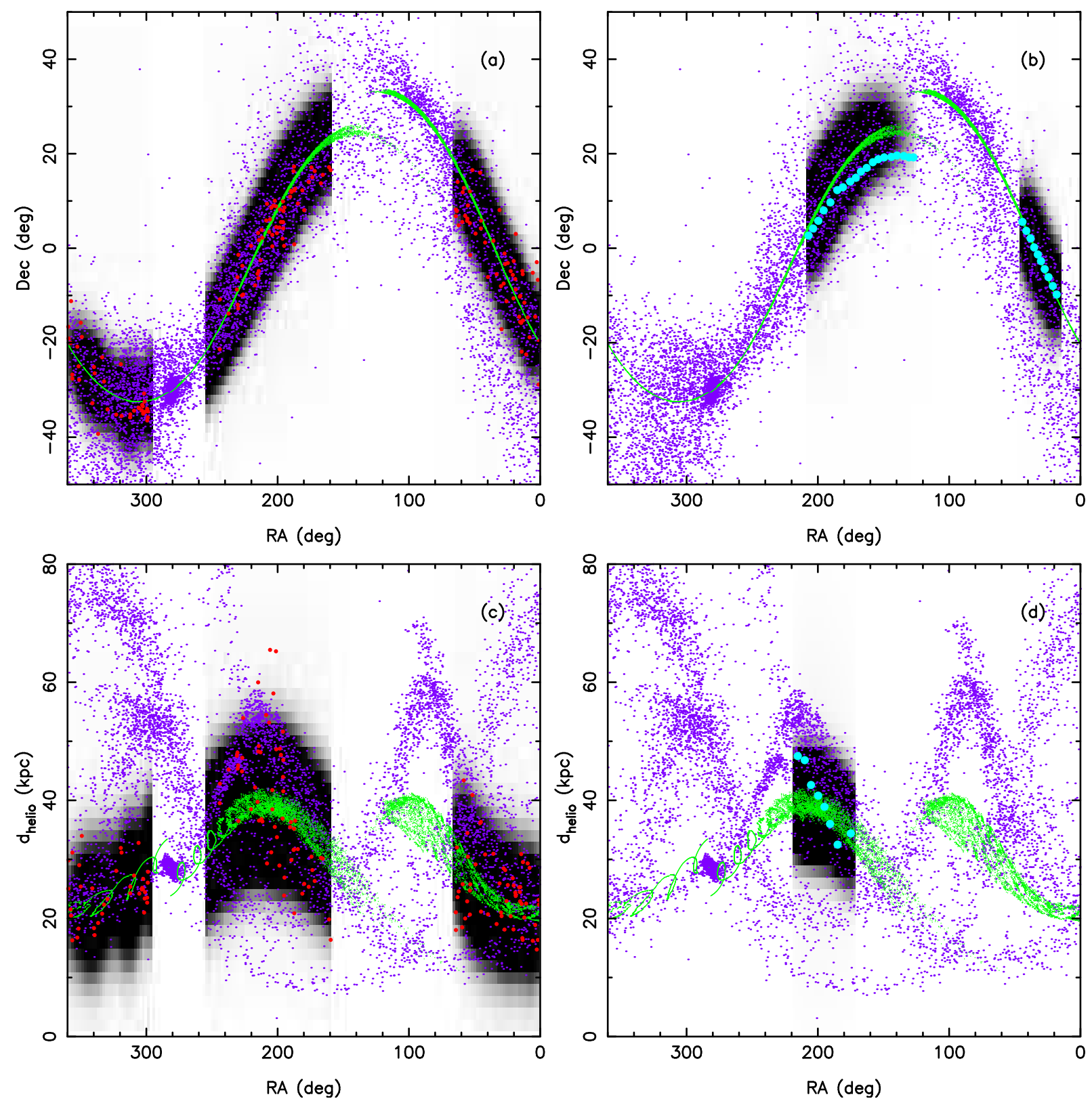

Figure 1. In panel (a), the sky positions in Right Ascension and Declination of the M-giant stars studied by LM10 (red circles) are plotted on top of the the best-fitting model of the Sgr stream found in $10^{6}$ iterations of the MCMC algorithm (green dots). The model points have been convolved with a Gaussian function to mitigate against sampling noise in the model, resulting in the probability density function map (black). Regions close to the Galactic plane have been masked out. Panel (b) shows the sky regions covered by the Sloan Digital Sky Survey (SDSS), where the stream is detected: our estimates of the SDSS sky positions of the main leading and trailing arms are shown with turquoise points. The model points that overlap these area are shown again in green. The lower panels show the corresponding distance profile, with (c) displaying the M-giants and (d) the distance estimates to red-clump stars in the SDSS as measured by Correnti et al. 2010. The fitted model can be seen to give a good approximation to all these data. The LM10 model (a random $10 \%$ selection of their particles) is shown in all panels with small purple dots.

method is able to provide a very close approximation to a stream without having to resort to N-body simulations. The simple insight behind this approximation is that a leading stream is formed from stars that leave the satellite close to the inner L1 Lagrange point, while the trailing stars leave close to the L2 Lagrange point. In this way, we avoid having to undertake a full N-body simulation, and can feasibly probe millions of model parameter combinations in an automatic way with a MCMC algorithm.

Following Dehnen \& Binney (1998, hereafter DB98), we model the Milky Way as a sum of density components, using the multipole expansion technique to solve the Poisson equation. For the bulge, disk, thick disk and interstellar medium component, we have adopted the parameter values of models ' $2 \mathrm{~b}$ ' and ' $2 \mathrm{c}$ ' from DB98: these are realistic density models that fit a wide range of observational constraints. DB98 fit these baryonic components within a halo component modeled as a double power law; the main difference between their models ' $2 b$ ' and ' $2 \mathrm{c}$ ' is that ' $2 \mathrm{~b}$ ' was forced to have an inner powerlaw exponent $\gamma_{h}=-2$, while model ' $2 \mathrm{c}$ ' had $\gamma_{h}=1$, 
similar to that of a universal (NFW) cosmological halo model (Navarro et al. 1997) (naturally, these constraints on the halo affect the fitted parameters of the other components). However, instead of adopting the DB98 double power-law dark matter halo model, we allow for a much larger amount of flexibility in the halo radial density profile, as we describe next. The halo is set to be an axisymmetric model, defined by a density at $10 \mathrm{kpc}$ together with an inner power law exponent $\gamma$ inside of $10 \mathrm{kpc}$, a density at $60 \mathrm{kpc}$ together with a power law exponent $\beta$ beyond that radius, and density values at 8 logarithmically-spaced radial locations between 10 and $60 \mathrm{kpc}$. A spline function is used to interpolate the density between these anchor points. Since the dynamics of the Sgr stream should not be very sensitive to the mass distribution in the inner Galaxy, or the distribution in the outer Galaxy beyond its apocenter, we decided to fix $\gamma=1$ and $\beta=3$, similar to the inner and outer behavior of an NFW halo model. However, the 10 halo density values are free parameters in our modeling. Although our code also allows one to set (or fit) the flattening in density $\left(q_{m}\right)$ of the axisymmetric halo component, for this particular experiment we chose to probe the case for a spherical halo, and so kept $q_{m}=1$ fixed.

The remaining parameters that need to be defined are the mass, 3-dimensional distance and velocity of Sgr as well as the position and velocity of the Sun, in order to correctly project the stream kinematics into line of sight observables.

For the initial analysis presented here, we chose $v_{\text {Sgr,helio }}=141 \mathrm{~km} \mathrm{~s}^{-1}$ (Bellazzini et al. 2008), and following LM10 we adopted $d_{S g r}=28 \mathrm{kpc}$, and $M_{S g r}=$ $2.5 \times 10^{8} \mathrm{M}_{\odot}$; these values were kept fixed. The total space velocity and direction of Sgr was allowed to vary, as were the Galactocentric radius of the Sun $R_{\odot}$ and the circular velocity of the Local Standard of Rest $v_{L S R}$. To be consistent with Majewski et al. (2004), we adopted their chosen Solar peculiar velocity of $(u, v, w)=$ $(-9,12,7) \mathrm{km} \mathrm{s}^{-1}$.

The MCMC algorithm that drives the simulation has been updated from that used in Varghese et al. (2011), and now uses an affine-invariant ensemblesampler scheme (with parallel tempering), identical to that described in Ibata et al. (2012). The algorithm starts off from an initial guess for the 14 model parameters (10 halo density values, 2 velocity parameters for Sgr, as well as $R_{\odot}$ and $\left.v_{L S R}\right)$, to begin its exploration of parameter space. Our initial estimations for the MCMC steps were adjusted dynamically in order to attain a target acceptance ratio of $25 \%$.

At each iteration, the algorithm makes a stream model that consists of a prediction of the locus of a tidal stream in space. We convert this to a probability density function by convolving the points by Gaussian functions that approximate the combined effect of the uncertainties on the observations (position, distance, velocity) and the expected intrinsic width of the stream. Finally, a likelihood for this set of parameter values is calculated from the product of the probabilities of the data (M-giant positions, distances and velocities; and SDSS field positions and distances) and the likelihood of the modelled Galactic rotation given the compilation by Sofue (2012).

\section{BEST-FIT MODEL}

The best-fit model in $10^{6}$ iterations of the above algorithm adopting the bulge, disk, thick disk and interstellar medium parameters of model '2c' of DB98 is shown in Figures 1] 3. Figure 1 displays the sky positions (top panels) and heliocentric distances (bottom panels) of the MCMC solution. The locus of the stream in these parameters is displayed in green, these are convolved with the parameter uncertainties and intrinsic stream width estimate to produce the probability density function displayed in the background grayscale image. For comparison we also display the LM10 triaxial N-body model (small purple dots). The left-hand panels show the positions of M-giant stars (red circles) from LM10 within $15^{\circ}$ of the orbital plane of the Sgr dwarf (Majewski et al. 2003); RA ranges close to the disk or close to the main body of the Sgr dwarf have been masked out. We discarded the region immediately surrounding Sgr as we wanted to avoid being affected by the dynamical properties of the progenitor (e.g. rotation). Panel (b) shows the sky position of the main detection of the Sgr stream in the SDSS (Belokurov et al. 2006; Koposov et al. 2012), as measured by us directly from SDSS data. Panel (d) shows the distance measurements of Correnti et al. (2010). In both right-hand panels we have masked out ranges in RA not probed by the data. It can be seen that the MCMC solution follows closely the position and distance constraints.

Figure 2 compares the radial velocity profile of the model to the M-giant data: the kinematic behavior is similar to that of the LM10 model, and reproduces well the trend in the M-giant radial velocities. The threedimensional structure of the data and models is shown in Figure 3, which gives an edge-on view of the Milky Way. As before, the red circles are the selection of LM10 M-giants, with SDSS red-clump distances from Correnti et al. (2010) shown with orange circles. The green dots mark the locus of the MCMC stream solution: the correspondence with the M-giant distances is not perfect in the leading arm, but neither is that of the LM10 N-body simulation (purple dots). Note that the M-giant distance uncertainties are considerable $(\sim 20 \% \sim$ Majewski et al. 2003), and there may also be a systematic difference with respect to the measurements based on the red clump or main sequence.

A comparison of the relative likelihood of our model versus that of LM10 is not very useful at this stage, the models are very different, and indeed both models fail to reproduce the data in detail (such as the stream bifurcation Belokurov et al. 2006), but it can be seen that in position, distance and velocity the two models are similar, and give a similarly good representation of the youngest parts of the leading and trailing arms.

While we use very similar constraints and assumptions to LM10, we have fit the Sgr stream without having to invoke the need for triaxiality. However, the price to pay for this is a peculiar radial mass distribution in the halo. Figure 4 shows the required rotation curve and corresponding density profile of the fitted spherical halo, again using the (fixed) non-halo components of model ' $2 \mathrm{c}$ ' from DB98. The red dots in panel (a) mark the measurements from Sofue (2012). Beyond the Solar Circle there is substantial uncertainty in the rotation curve, as the large scatter reveals, and beyond $20 \mathrm{kpc}$ there are virtually no direct constraints. Expectations from $\Lambda$-CDM would 


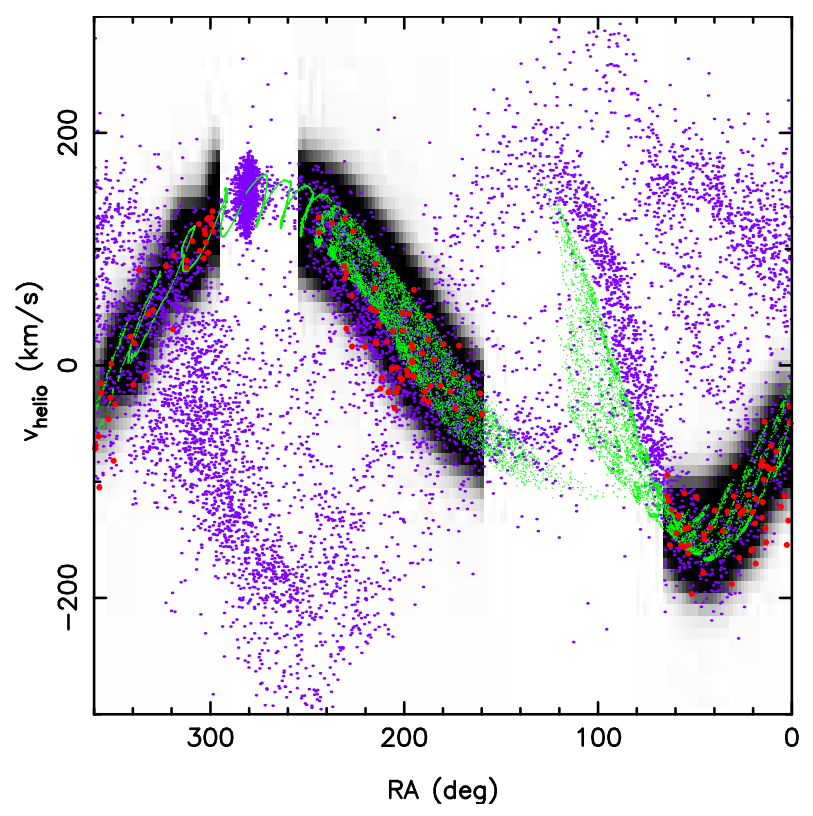

Figure 2. The heliocentric velocities of the LM10 M-giants (red circles) are compared to the best-fitting model (green points). The correspondence can be seen to be as good as that of LM10.

suggest that the rotation curve should decline slowly beyond $10 \mathrm{kpc}$ (Klypin et al. 2002), in stark contrast to our model's requirement. Nevertheless, we note that HI observations of our neighboring galaxy, Andromeda (black dots in panel ' $a$ ' - Chemin et al. 2009), show a rotation curve rise beyond $25 \mathrm{kpc}$ that is similar to our model.

While it was not our intention to attempt to fit an accurate mass model of the inner Galaxy, it is worth noting that the surface mass density of the disk and the vertical force $K_{z}$ lie within observationally-acceptable ranges (see DB98). In addition, the Oort constants for our model (which are not identical to those of DB98 since we have altered the halo) are $A=13.8$ and $B=-13.5$, close to the observed $A=14 \pm 0.8, B=-12.4 \pm 0.6$ (see Table 3 of DW12 for comparison to other solutions). The detailed structure of the mass distribution in the inner Galaxy turns out to be relatively unimportant in fitting the Sgr stream: if we use the non-halo components of model ' $2 \mathrm{~b}$ ' from DB98 (instead of those of ' $2 \mathrm{c}$ ' as in Figures 1-4) the MCMC algorithm also converges on similar solutions that require a rising rotation curve.

\section{DISCUSSION AND CONCLUSIONS}

The analysis we have presented here shows that it is possible to reproduce the spatial and kinematic structure of the Sgr stream at approximately the same level of precision as LM10 without the need for a triaxial halo, if the Galactic rotation curve rises significantly in the range $\sim 20 \mathrm{kpc}$ to $\sim 60 \mathrm{kpc}$. Interestingly, the break in the density profile occurs at approximately where the outer stellar halo component proposed by Carollo et al. (2007) becomes dominant. This rotation curve rise of course places a substantial amount of matter in the outer Galaxy, which has the effect of braking the leading arm, bringing its velocity into agreement with that of the Mgiants, particularly in the region around $R A \sim 200^{\circ}$. In addition to the MCMC parameter survey discussed above, we have also attempted an interactive investigation, choosing parameter combinations judiciously; how-

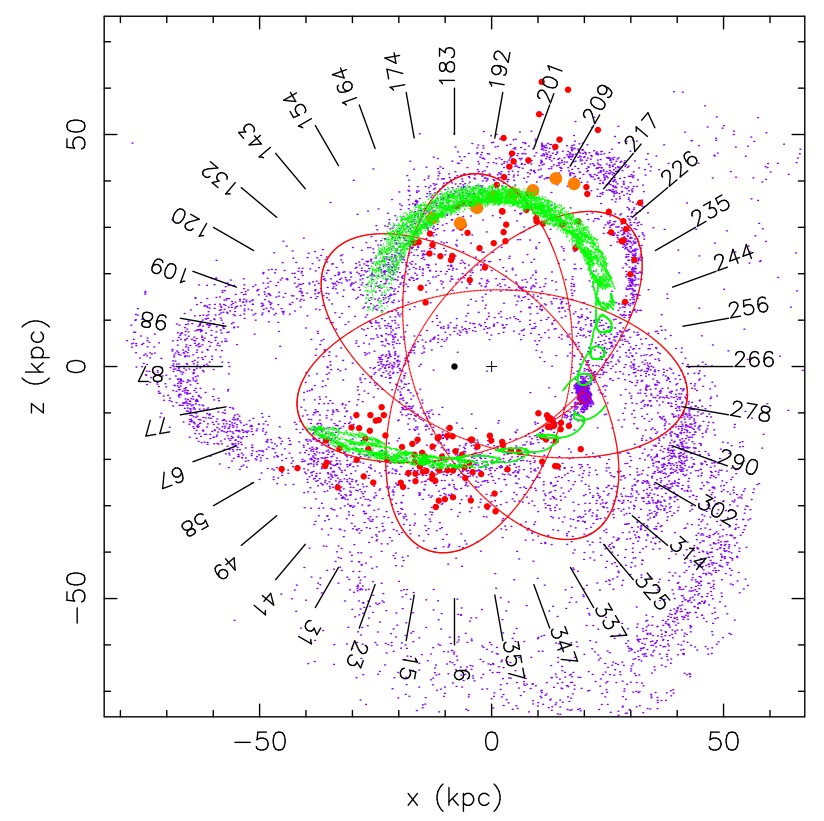

Figure 3. An $x-z$ cut through the Milky Way, showing the stream location (green dots) according to our best-fit model. The path of the centre of mass of the Sgr dwarf is shown in red; the fact that this does not line up with the stream position is due to the self-gravity of the dwarf galaxy, which we take into account in our modeling, but is neglected in the DW12 analysis. The LM10 M-giants are shown with red circles, and the big orange dots mark the Correnti et al. 2010 'A'-field measurements from the SDSS. The small cross at the origin mark the Galactic Centre, and the position of the Sun is shown with a black circle at $(-8,0) \mathrm{kpc}$. The numbers marked on the circular ticks are the corresponding Right Ascension values (in deg) in the plane of the Sgr stream.

ever, we were unable to find another acceptable solution without a strong rotation curve rise.

It is unclear at present what the structure of galactic mass dark matter halos actually is in nature, but in $\Lambda$-CDM, dark matter density profiles decline as $r^{-2}$ only when averaged over a very large number of halos. The profile of Fig. $4 \mathrm{~b}$ is therefore not inconsistent with $\Lambda$-CDM, and indeed a rotation curve rise of similar magnitude is observed in M31 over a similar radial range (Fig. 4h). We suspect (although we have not modeled this) that there is a trade-off between triaxiality and rotation curve rise, so that a less triaxial model than that of LM10 (which is actually an oblate spheroid oriented perpendicular to the Galactic disk) can be fitted with a less extreme rotation curve than that presented here.

A consequence of this new solution is that the Milky Way would be considerably more massive than estimated from models with a falling rotation curve within $R<$ $60 \mathrm{kpc}$. Assuming a virial radius of $258 \mathrm{kpc}$ Klypin et al. 2002), leads to a virial mass of $2.6-3.1 \times 10^{12} \mathrm{M}_{\odot}$ for our model. It is interesting to note that the LMC and SMC would clearly be bound to the Milky Way if this reflects the true mass of our Galaxy, contrary to conclusions based on HST proper motion measurements that assume a standard Galactic mass model (see.e.g., Besla et al. 2007). To be consistent with the timing argument limit for the mass of the Local Group of $5.58_{-0.25}^{+0.26} \times 10^{12} \mathrm{M}_{\odot}$ (van der Marel \& Guhathakurta 2008), the dark matter would have to be primarily concentrated within the two giant galaxies. While the above mass for the Milky Way is certainly substantially higher than most recent esti- 

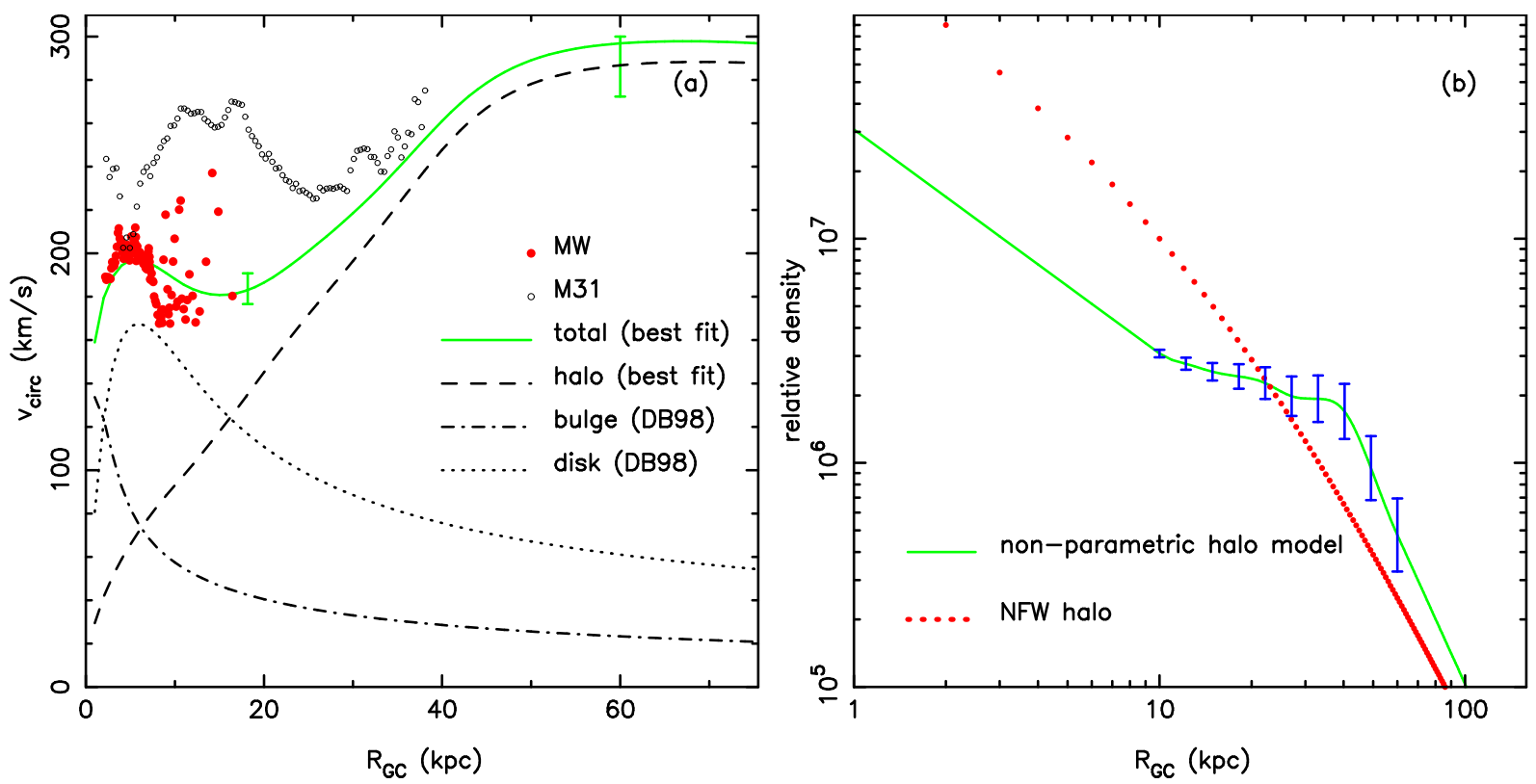

Figure 4. The rotation curve required by our non-parametric spherical halo model is shown with a solid line in panel (a), with its decomposition into halo (dashed line), bulge (dot-dashed line) and disk (dotted line) components. This rotation curve fits the available measurements of Sofue (2012) (red dots), but it requires significantly rising values beyond $\sim 30 \mathrm{kpc}$. Although such a rising rotation curve is unexpected, it is similar to the observed HI rotation curve of the Andromeda galaxy (black circles — Chemin et al. 2009). The two error bars at $R_{G C}=18 \mathrm{kpc}$ and $60 \mathrm{kpc}$ show the $1 \sigma$ uncertainties derived from the MCMC algorithm (note that a prior impedes solutions with $v_{\text {circ }}>300 \mathrm{~km} \mathrm{~s}^{-1}$ ). Panel (b) shows the corresponding density profile (solid line). The error bars mark the radial location of the density anchor points together with the $1 \sigma$ spread from the MCMC fit. For reference we also show an NFW profile (dots) of scale radius $21.5 \mathrm{kpc}$ (Klvpin et al. 2002): while this has an identical asymptotic behavior to the halo model shown, it is substantially different in the radial range probed by the Sgr stream.

mates (e.g., Watkins et al. 2010), almost all the mass constraints at large distance are based on Jeans equations solutions that make the unjustified assumptions of statistical independence of the halo tracers and some form for the orbital anisotropy.

In summary, we present this solution as a means to avoid the triaxial halo models of LM10 or DW12, and in particular their peculiar orientation as oblate structures perpendicular to the Galactic plane. We recognize of course that the solution presented here is also strange, being spherical and possessing a very high mass, but we judge that it is not ruled out by observational constraints. The significant shortcomings of both the LM10/DW12 and our models suggest that they do not include sufficient of the actual complexity of (probably both) Sgr and the Milky Way halo. Future modeling of the more ancient parts of the Sgr stream may help discriminate between these models, and pave the way to better solutions.

R.A.I. gratefully acknowledges support from the Agence Nationale de la Recherche though the grant POMMME (ANR 09-BLAN-0228). GFL thanks the Australian Research Council for support through his Future Fellowship (FT100100268) and Discovery Project (DP110100678)

\section{REFERENCES}

Allgood B., Flores R. A., Primack J. R., Kravtsov A. V., et al. , 2006, MNRAS, 367, 1781
Bellazzini M., Ibata R. A., Chapman S. C., et al. , 2008, AJ, 136, 1147

Belokurov V., Zucker D. B., Evans N. W., et al. , 2006, ApJ, 642, L137

Besla G., Kallivayalil N., Hernquist L., et al. , 2007, ApJ, 668, 949 Carollo D., Beers T. C., Lee Y. S., et al. , 2007, Nature, 450, 1020 Chemin L., Carignan C., Foster T., 2009, ApJ, 705, 1395

Correnti M., Bellazzini M., Ibata R. A., Ferraro F. R., Varghese A., 2010, ApJ, 721, 329

Debattista V. P., Moore B., Quinn T., et al. , 2008, ApJ, 681, 1076

Deg N., Widrow L., 2012, MNRAS, 428, 912

Dehnen W., Binney J., 1998, MNRAS, 294, 429

Ibata R., Nipoti C., Sollima A., et al. , 2012, MNRAS, 225

Ibata R., Lewis G. F., Irwin M., Totten E., Quinn T., 2001, ApJ, 551,294

Klypin A., Zhao H., Somerville R. S., 2002, ApJ, 573, 597

Komatsu E., Smith K. M., Dunkley J., et al. , 2011, ApJS, 192, 18

Koposov S. E., Belokurov V., Evans N. W., et al. , 2012, ApJ, 750,80

Law D. R., Majewski S. R., 2010, ApJ, 714, 229

Majewski S. R., Kunkel W. E., Law D. R., et al. , 2004, AJ, 128, 245

Majewski S. R., Skrutskie M. F., Weinberg M. D., Ostheimer J. C., 2003, ApJ, 599, 1082

Navarro J. F., Frenk C. S., White S. D. M., 1997, ApJ, 490, 493

Sofue Y., 2012, PASJ, 64, 75

van der Marel R. P., Guhathakurta P., 2008, ApJ, 678, 187 Varghese A., Ibata R., Lewis G. F., 2011, MNRAS, 417, 198 Watkins L. L., Evans N. W., An J. H., 2010, MNRAS, 406, 264 